\title{
Survivin expression in ovarian cancer and its correlation with clinico-pathological, surgical and apoptosis-related parameters
}

\author{
G Ferrandina ${ }^{*}, 1$, F Legge', E Martinelli', FO Ranelletti ${ }^{2}$, GF Zannoni ${ }^{3}$, L Lauriola ${ }^{3}$, M Gessi $^{3}$, V Gallotta' and \\ G Scambia ${ }^{4}$
}

'Gynecologic Oncology Unit, Catholic University of the Sacred Heart, Largo A. Gemelli, 8, 00168 Rome, Italy; ${ }^{2}$ Department of Histology, Catholic University of the Sacred Heart, Rome, Italy; ${ }^{3}$ Department of Pathology, Catholic University of the Sacred Heart, Rome, Italy; ${ }^{4}$ Department of Oncology, Catholic University of the Sacred Heart, Campobasso, Italy

\begin{abstract}
We investigated the association of survivin expression with prognosis and other apoptosis-related biological factors in II 0 primary ovarian cancer patients admitted to the Division of Gynecologic Oncology, Catholic University of Rome. Immunohistochemistry was performed on formalin-fixed, paraffin-embedded sections by using polyclonal antibody ab469 for survivin, and mouse monoclonal antibodies (clone 124 and DO-7), for bcl-2 and p53, respectively. Cytoplasmic survivin immunoreaction was observed in $84.5 \%$ cases, while nuclear survivin immunostaining was observed in $29.1 \%$ cases. We failed to find any relationship between cytoplasmic survivin positivity rate and any of the parameters examined. Serous tumours showed a lower percentage of nuclear survivin positivity with respect to other histotypes $(20.5$ vs $48.6 \%$, respectively; $P$-value $=0.004)$. The percentage of nuclear survivin positivity was higher in cases subjected to primary tumour cytoreduction (43.5\%), with respect to patients subjected to exploratory laparotomy (20\%) $(P=0.024)$. Bcl-2 and p53 were, respectively, expressed in 27.3 and $60.0 \%$ of the cases and their expression was not correlated with survivin status. During the follow-up period, progression and death of disease were observed in 68 (61.8\%) and 53 (48.2\%) cases, respectively. There was no difference in time to progression and overall survival according to survivin status in ovarian cancer patients. In conclusion, in our experience, the immunohistochemical assessment of survivin status does not seem to be helpful in the prognostic characterisation of ovarian cancer. A more in depth investigation of the complex physiology of divergent survivin variants is needed in order to clarify the biological and the clinical role of differentially located survivin isoforms.
\end{abstract}

British Journal of Cancer (2005) 92, 27I-277. doi:I0.1038/sj.bjc.6602332 www.bjcancer.com

Published online 18 January 2005

(C) 2005 Cancer Research UK

Keywords: ovarian cancer; survivin; prognosis

Although several clinicopathological parameters have been reported to be of prognostic significance in ovarian cancer, including F.I.G.O. stage, volume of residual disease, presence of cytologically malignant ascites and grade of tumour differentiation (Hoskins et al, 2000), it is conceivable that the assessment of biochemical factors more strictly related to tumour cell biology and intrinsic aggressiveness could help identifying high-risk patients and facilitating management of this disease.

Apoptosis (programmed cell death) has been proposed to play a role not only in cancer onset and progression but also in sustaining decreased tumour cell sensitivity to chemotherapy (Hickman, 1992; Thompson, 1995), which still represents one of the main prognostic indicators in this neoplasia (Hoskins et al, 2000). In this context, the analysis of the molecules possibly involved in the modulation of apoptosis seems to be particularly attractive.

Survivin is a member of the inhibitors of apoptosis protein (IAP) family, which participates in the complex network regulating programmed cell death and also cell division (Ambrosini et al,

*Correspondence: Dr G Ferrandina; E-mail: gabriella.ferrandina@libero.it Received 21 July 2004; revised 20 September 2004; accepted II November 2004; published online 18 January 2005
1997; Altieri and Marchisio, 1999; Salvesen and Duckett, 2002). Survivin protein is commonly detected in fetal tissues but not in normal adult tissues, while being overexpressed in human cancer, thus suggesting the contribution of survivin gene reactivation in carcinogenesis (Ambrosini et al, 1997). The crucial role of survivin in protection from apoptosis is supported by the observations that forced expression of survivin counteracts cell death induced by several apoptotic stimuli (Li et al, 1998), while survivin inhibition by antisenses causes spontaneous apoptosis (Olie et al, 2000). Survivin activity seems to be mediated by the inhibition of caspase pathways and also interaction with the microtubules of the mitotic spindle during the G2/M phase of cell cycle (Deveraux and Reed, 1999; Li, 2003), which suggest that survivin could promote tumour growth by dual functions, that is, inhibition of apoptosis and induction of mitogenesis. Overexpression of survivin has been associated with parameters of aggressiveness and poor prognosis in several solid tumours, although conflicting data have also been reported $(\mathrm{Li}, 2003)$. As far as ovarian lesions are concerned, a higher percentage of survivin overexpression was found in borderline and malignant tumours with respect to benign lesions (Sui et al, 2002).

Several studies have attempted to delineate the clinical role of survivin expression in epithelial ovarian carcinomas but no definitive conclusions could be drawn probably because of the 
size and/or heterogeneity of the population series, incompleteness of clinical informations as well as the use of different antibodies and methods of score of survivin expression (Yoshida et al, 2001; Sui et al, 2002; Takai et al, 2002; Zaffaroni et al, 2002; Cohen et al, 2003).

The aim of this study was to investigate the association of survivin expression with surgical data, response to chemotherapy and prognosis in a series of primary ovarian cancer patients undergoing surgery, chemotherapy treatment, and follow-up performed by the same gynaecological oncology team. Moreover, considering the biological relevance of the apoptosis regulatory proteins such as p53 and bcl-2 in the complex regulation of apoptosis (Miyashita and Reed, 1993; Miyashita et al, 1994), the association of survivin with these biological parameters has been also investigated.

\section{MATERIALS AND METHODS}

\section{Patients}

The study included 110 primary ovarian cancer patients admitted to the Division of Gynecologic Oncology, Catholic University of Rome between May 1986 and September 2002. The median age was 58.5 years (range $25-84)$. A total of $91(82.7 \%)$ patients were stage III-IV. The other clinicopathological characteristics are listed in Table 1. According to the standard guidelines for ovarian cancer primary treatment, maximal surgical effort has been attempted in all patients resulting in successful debulking in 65 (59.1\%) cases, which were subjected to surgical removal of tumour masses, along with total abdominal hysterectomy, adnexectomy, radical omentectomy, appendectomy and multiple biopsies, and additional surgery (intestinal resection, diaphragm stripping) when required. Radical pelvic and para-aortic lymphadenectomy was performed in all patients undergoing primary cytoreduction who had absent or microscopic residual disease or residual tumour less than $2 \mathrm{~cm}$.

Table I Patient characteristics

\begin{tabular}{lc}
\hline Characteristics & No. of patients (\%) \\
\hline All cases & 110 \\
Age (years) & $56(50.9)$ \\
$\quad<60$ & $54(49.1)$ \\
$\geqslant 60$ & \\
Ascites & \\
No & $47(42.7)$ \\
Yes & $63(57.3)$ \\
Histotype & \\
Serous & \\
Mucinous & $73(66.4)$ \\
Endometrioid & $4(3.6)$ \\
Undifferentiated & $21(19.1)$ \\
Clear cell & $7(6.4)$ \\
n.a. & $3(2.7)$ \\
Grade & $2(1.8)$ \\
GI -2 & \\
G3 & \\
n.a. & $26(23.4)$ \\
FIGO stage & $74(67.3)$ \\
I-II & $10(9.3)$ \\
III-IV & \\
Response to chemotherapy ${ }^{\mathrm{a}}$ & \\
Complete/partial & $19(17.3)$ \\
No change/progression & $91(82.7)$ \\
\hline Ony FIGO stage II-IV. & \\
&
\end{tabular}

aOnly FIGO stage III-IV.
In total, 45 (40.9\%) cases were judged to be unresectable at first surgery because of extensive peritoneal bulky carcinomatosis, agglutinated bowel/mesentery and infiltration of the upper gastrointestinal tract and/or the major vessels (Fanfani et al, 2003), and were submitted only to multiple biopsies.

Patients cytoreduced at first surgery received 4-6 cycles of chemotherapy 2-3 weeks after primary surgery, unless they showed clinical progression during treatment. As far as patients undergoing explorative laparotomy are concerned, they received three or four cycles of neoadjuvant chemotherapy before attempting a second cytoreductive surgery, unless they showed clinical progression during treatment. All patients underwent platinumbased chemotherapy (cisplatin: $75-100 \mathrm{mg} \mathrm{m}^{-2}$ for each cycle or carboplatin 5 AUC, q21), including also paclitaxel in $61(58.6 \%)$ cases.

Response to chemotherapy was assessed by gynaecological exam, ultrasound examination, analysis of CA125 levels and CT scan, if necessary, and was recorded according to World Health Organization criteria (1979). In the subgroup of patients who were not susceptible to be cytoreduced at first surgery, a direct assessment of response to chemotherapy was carried out in case of clinical response, at the time of second laparotomy.

\section{Immunohistochemistry}

Tumour tissues' biopsies from primary tumours were obtained at first surgery in all cases. Tissue specimens were fixed in formalin and paraffin-embedded according to standard procedures. In all, $3 \mu \mathrm{m}$ of representative blocks from each case were deparaffinised in xylene, rehydrated, treated with $3 \% \mathrm{H}_{2} \mathrm{O}_{2}$ in TBS for $5 \mathrm{~min}$ to block endogenous peroxidase activity, and subjected to heatinduced epitope retrieval in microwave oven using $10 \mathrm{~mm}$ citric acid at $\mathrm{pH}$ 6.0. Sections were incubated with normal goat serum $20 \%$ for $30 \mathrm{~min}$, then with polyclonal antibody ab496 (ABCAM Limited, Cambridge, UK) diluted according to the manufacturer's instructions, overnight. Bcl-2 and p53 were analysed using mouse monoclonal antibodies (clone 124 and DO-7, respectively, DAKO, Carpintera, CA, USA), according to the methods previously described (Ferrandina et al, 1999; Ferlini et al, 2003).

Slides from all cases studied were then simultaneously processed for immunohistochemistry using En Vision-rabbit + System-HRP DAKO (Carpintera, CA, USA). Diaminobenzidine was used as a chromogen (DAB substrate System, DAKO). Negative controls were performed using nonimmunised rabbit serum or by omitting the primary antiserum.

The analysis of all tissue sections was carried out without any prior knowledge of the clinical parameters by three authors (MG, LL, FL) by means of light microscopy. Proportion of immunostained cells was scored at low magnification $(\times 5$ objective lens $)$ by evaluating the entire tumour area. The intensity of staining (scale $0-4)$ and the percentage of stained cells were evaluated. The following cutoff were ' $a$ priori' chosen for scoring: cases with more than $20 \%$ of cells showing intensity of cytoplasmic staining $>1$ were considered positive for cytoplasmic survivin expression, while cases with nuclear staining $\geqslant 1$ in more than $5 \%$ of cells were considered as nuclear survivin positive. In case of disagreement $(n=13,11.8 \%)$, sections were submitted to a rejoint evaluation.

\section{Statistical analysis}

Fisher's exact test or $\chi^{2}$ test were used to analyse the distribution of surviving-positive cases according to clinicopathological, surgical, and biological features.

Overall survival (OS) and time to progression (TTP) were calculated from the date of diagnosis to the date of death/ progression or date last seen. Medians and life tables were computed using the product-limit estimate by the Kaplan and Meier method (1958), and the log-rank test was employed to assess 
the statistical significance (Mantel, 1966). Statistical analysis was carried out using SOLO (BMDP Statistical Software, Los Angeles, CA, USA).

\section{RESULTS}

\section{Survivin immunostaining}

As shown in Figure 1, specific survivin immunostaining was observed both in the cytoplasm and nuclear compartment (A) of tumour cells, or only in the cytoplasm (B) or nucleus (C).

Cytoplasmic survivin immunoreaction was observed in 93 $(84.5 \%)$ cases, while nuclear survivin immunostaining was observed in only $32(29.1 \%)$ cases: $29(26.4 \%)$ cases showed survivin staining both in nuclear and cytoplasmic compartment.

There seems to be no association between cytoplasmic and nuclear survivin staining; however, nine out of $13(69.2 \%)$ cases with very strong cytoplasmatic intensity $($ score $=4)$ showed also nuclear staining in contrast with only 19 out of $80(23.7 \%)$ cases with lighter $($ score $=2,3)$ cytoplasmic staining $(P=0.002)$.

\section{Correlation with clinical, surgical and pathological parameters}

Table 2 shows the distribution of cytoplasmic and nuclear survivin positivity according to clinicopathological characteristics. As far as cytoplasmic survivin reaction is concerned, we failed to find any relationship between positivity rate and any of the clinicopathological parameters examined. As far as nuclear survivin expression is concerned, serous tumours showed a lower percentage of nuclear survivin positive immunoreaction with respect to other histotypes (20.5 vs $48.6 \%$, respectively; $P$-value $=0.004)$. On the other hand, nuclear survivin staining was not differently distributed according to other clinical-pathological parameters. No association between survivin status and response to chemotherapy was found. Moreover, neither nuclear nor cytoplasmic survivin staining were found to be associated with response to treatment in subgroups of patients receiving platinum-based $v s$ paclitaxel-containing chemotherapy (data not shown).

Table 3 shows the distribution of cytoplasmic and nuclear survivin expression according to surgical features in stage III-IV ovarian cancer patients.

Cytoplasmic survivin immunostaining was not differently distributed according to any of the parameters analysed.

On the other hand, the percentage of nuclear survivin positivity was higher in cases in which tumour cytoreduction was achieved at primary surgery (20 out of $46,43.5 \%$ ) with respect to patients subjected to exploratory laparotomy (nine out of $45,20 \%$ ) $(P=0.024)$. Nuclear survivin staining was found not to be different according to the extent of debulking since nuclear survivin positivity was found in seven out of $16(43.7 \%)$ cases achieving optimal cytoreduction (residual tumour $<0.5 \mathrm{~cm}$ ) vs 13 out of 30 $(43.3 \%)$ patients achieving suboptimal (residual tumour $\geqslant 0.5 \mathrm{~cm}$ ) debulking $(P$-value $=$ n.s. $)$.

We found a higher percentage of nuclear survivin positivity in patients with no apparent mesenteric infiltration (24 out of 60 , $40.0 \%$ ) with respect to patients with tumour mesenteric involvement $(5$ out of $31,16.1 \%, P$-value $=0.017)$. In addition, the percentage of nuclear survivin positivity was also higher in cases without spread of disease in the upper region of the abdomen $(52.2 \%)$ with respect to patients with this feature $(24.3 \%$, $P$-value $=0.014)$.

\section{Correlation with apoptosis-related parameters}

Bcl-2 and p53 expression was evaluated in 80 cases. Bcl-2 and p53 were expressed in 27.3 and $60.0 \%$, respectively, of the cases examined. As shown in Table 4, neither cytoplasmic or nuclear survivin immunostaining were differently distributed according to any of the apoptosis-related parameters analysed.

\section{Survival analysis}

Follow-up data were available for 110 patients. As of May 2004, the median follow up was 32 months (range 1-221). During the
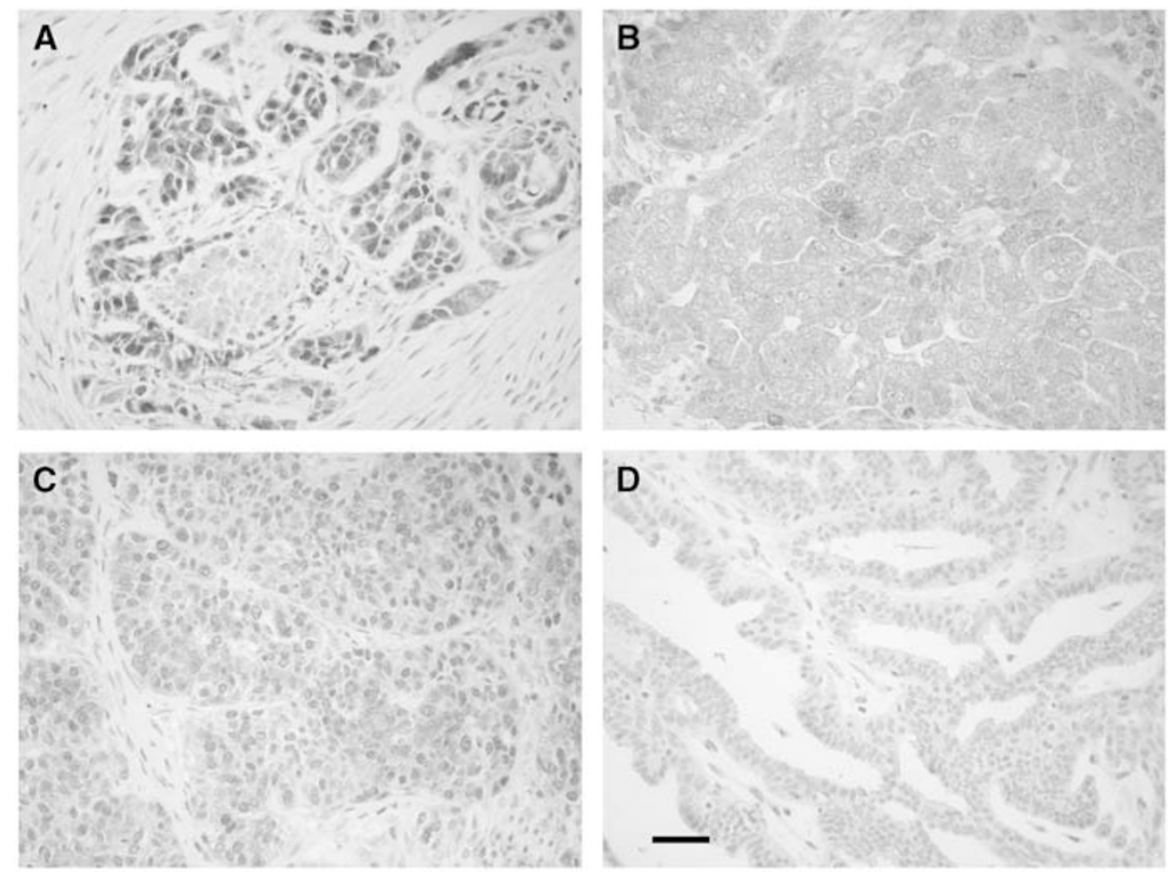

Figure I Survivin immunostaining in primary ovarian cancer. Specific survivin immunostaining was observed in both the cytoplasm and the nuclear compartment $(\mathbf{A})$, either in the cytoplasm only (B), or in nuclear compartment only (C). An example of surviving-negative ovarian tumour (D). $\operatorname{Bar}=35 \mu \mathrm{m}$. 
Table 2 Nuclear and cytoplasmic survivin expression according to clinical and pathological characteristics in ovarian cancer

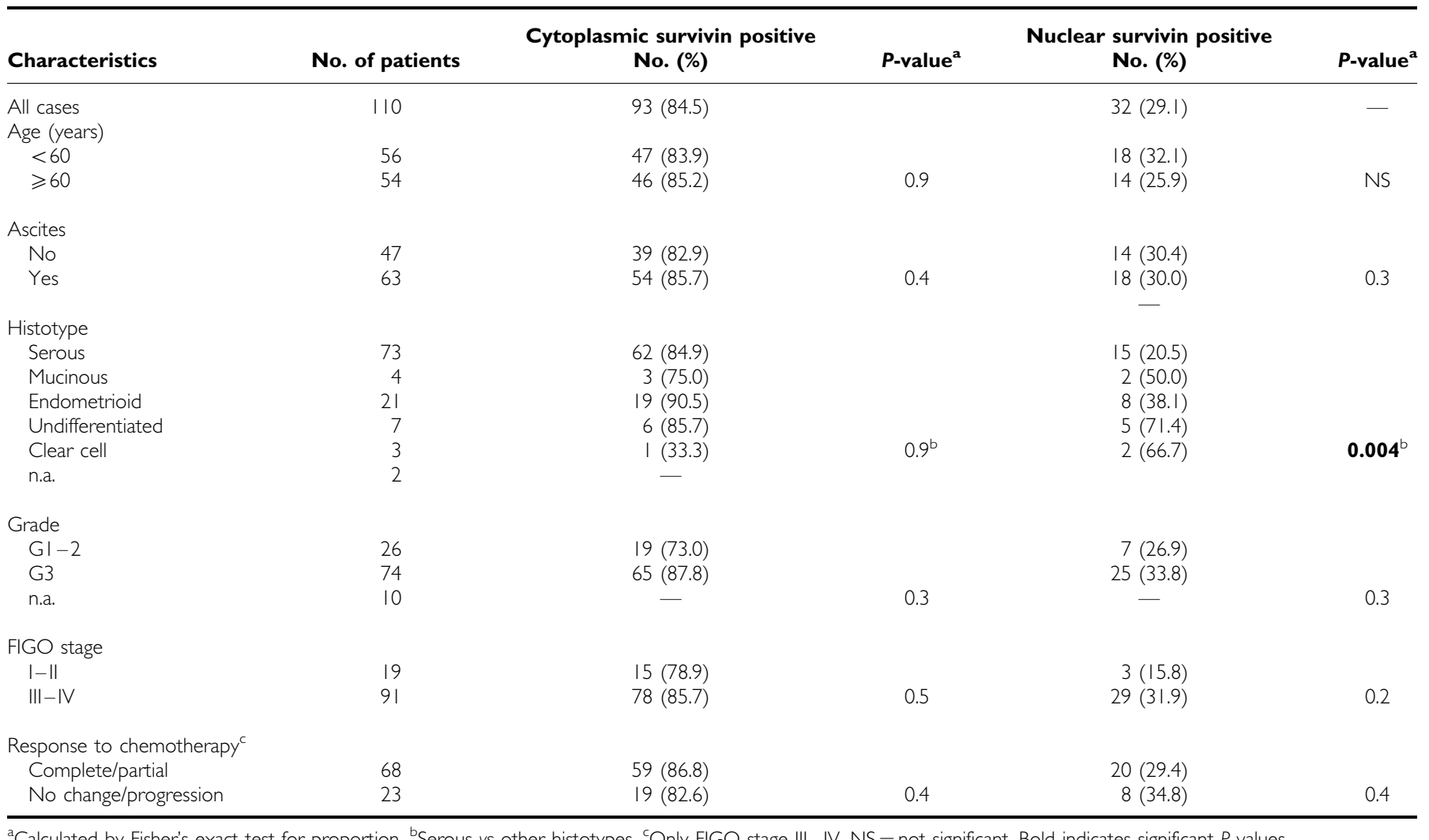

Table 3 Nuclear and cytoplasmic survivin expression according to surgical parameters in stage III-IV ovarian cancer

\begin{tabular}{|c|c|c|c|c|c|}
\hline All cases & 91 & $78(85.7)$ & & $29(31.9)$ & - \\
\hline Cytoreduction & 46 & $39(84.8)$ & & $20(43.5)$ & \\
\hline Explorative laparotomy & 45 & $39(86.7)$ & 0.9 & $9(20.0)$ & 0.024 \\
\hline \multicolumn{6}{|l|}{ Carcinomatosis } \\
\hline \multicolumn{6}{|l|}{ Mesenteric infiltration } \\
\hline No & 60 & $52(86.7)$ & & $24(40.0)$ & \\
\hline Yes & 31 & $26(83.9)$ & 0.5 & $5(16.1)$ & 0.017 \\
\hline \multicolumn{6}{|l|}{ Frozen pelvis } \\
\hline No & 61 & $52(85.2)$ & & $18(29.5)$ & \\
\hline \multirow[t]{2}{*}{ Yes } & 23 & $19(82.6)$ & & $12(52.2)$ & \\
\hline & 70 & $59(84.3)$ & 0.5 & $17(24.3)$ & 0.014 \\
\hline
\end{tabular}

${ }^{a}$ Calculated by Fisher's exact test for proportion. Bold indicates significant $P$-values.

follow-up period, progression and death of disease were observed in $68(61.8 \%)$ and $53(48.2 \%)$ cases, respectively.

As shown in Figure 2, there was no difference in TTP according to cytoplasmic and nuclear survivin status in ovarian cancer patients. Similar results were observed when considering the OS curves (data not shown).

\section{DISCUSSION}

Despite the availability of several studies exploring the expression of survivin protein in primary ovarian cancer, no definitive conclusions have been provided about its possible clinical role in this neoplasia (Yoshida et al, 2001; Sui et al, 2002; Takai et al, 2002; 


\begin{tabular}{|c|c|c|c|c|c|}
\hline Characteristics & No. of patients & $\begin{array}{c}\text { Cytoplasmic survivin positive } \\
\text { No. (\%) }\end{array}$ & $P$-value ${ }^{a}$ & $\begin{array}{c}\text { Nuclear survivin positive } \\
\text { No. (\%) }\end{array}$ & $P$-value ${ }^{a}$ \\
\hline \multicolumn{6}{|l|}{ Bcl-2 status } \\
\hline Negative & 58 & $48(82.7)$ & & $23(9.6)$ & \\
\hline Positive & 22 & $17(77.3)$ & NS & $7(31.8)$ & NS \\
\hline \multicolumn{6}{|l|}{ P53 status } \\
\hline Negative & 32 & $28(87.5)$ & & $9(28.1)$ & \\
\hline Positive & 48 & 41 (85.4) & NS & $14(29.2)$ & NS \\
\hline
\end{tabular}

${ }^{a}$ Calculated by Fisher's exact test for proportion. NS = not significant.
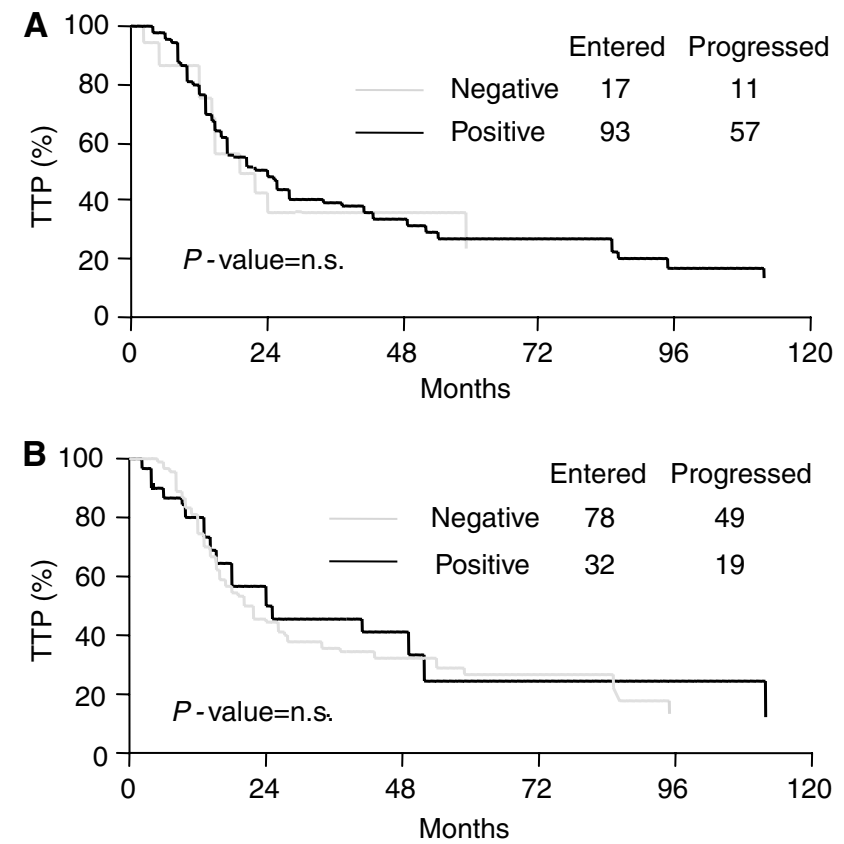

Figure 2 Time to progression (TTP) curves according to cytoplasmic (A) and nuclear (B) survivin status in ovarian cancer.

Zaffaroni et al, 2002; Cohen et al, 2003). As emphasised in Table 5, discrepancies among studies can be explained by size and clinical characteristics of the series examined (Yoshida et al, 2001; Sui et al, 2002; Takai et al, 2002; Cohen et al, 2003), as well as methodological differences: indeed, different antibodies as well as scoring systems for evaluation of survivin immunoreaction have been utilised. Moreover, survivin staining has been described as prevalent either in the cytoplasm (Yoshida et al, 2001; Takai et al, 2002; Zaffaroni et al, 2002) or in the nucleus (Sui et al, 2002; Takai et al, 2002; Cohen et al, 2003), and it has been not always clearly stated whether the overall rate of positivity was derived from a single subcellular compartment or both.

We first separately describe the expression of survivin protein in cytoplasmic and nuclear compartments of ovarian tumour cells. With the use of ab496 antibody, survivin was shown to be predominantly detected in the cytoplasmic compartment of ovarian cancer cells, confirming previous observations obtained with the same antibody after subcellular fractionation of HeLa cells (Fortugno et al, 2002). In particular, Fortugno et al (2002) showed that cytoplasmic and nuclear survivin pools are immunochemically different and this might partly explain the conflicting data on survivin localisation in solid tumours (Li, 2003). Besides, cytoplasmic and nuclear survivin are independently modulated during cell cycle progression and only cytoplasmic survivin associates with $\mathrm{p} 34^{\mathrm{cdc} 2}$ and is phosphorylated on Thr34, event which seems to mediate the antiapoptotic function of the protein (O'Connor et al, 2000), thus suggesting that the two pools exert different biological functions. Moreover, data have been provided about the existence of functionally divergent survivin splice variants, which exhibit different subcellular localisations (Mahotka et al, 2002).

The relationship between immunohistochemically detected cytoplasmic and nuclear localisation of survivin has been not investigated: although we failed to find any relationship between cytoplasmic and nuclear staining, a higher percentage of cases with very strong cytoplasmic intensity showed also nuclear survivin immunoreaction. Further studies aimed at clarifying the functional relationship between cytoplasmic and nuclear survivin are needed in order to properly assess the role of immunohistochemically detected survivin pools in human cancer.

As far as the association between survivin and clinicopathological and surgical parameters is concerned, we showed that serous ovarian tumours expressed a significantly lower nuclear survivin content with respect to other histotypes, as also reported by Yoshida et al (2001). Moreover, an intriguing association between high expression of nuclear survivin and better chance of performing tumour cytoreduction at first surgery was shown, which is likely to be supported by the association of higher nuclear survivin content with the absence of distinctive patterns that usually preclude the feasibility of cytoreduction, such as tumour involvement of upper abdominal organs and mesentery (Fanfani et al, 2003). Our data are unlikely to be influenced by the initial tumour extension, since no correlation between survivin expression and stage of disease was found, as previously reported (Sui et al, 2002; Zaffaroni et al, 2002). However, the association between high nuclear survivin expression and better chance of cytoreduction, which represents one of the major determinants of response to chemotherapy and favourable prognosis (Hoskins et al, 2000), does not seem to translate into major differences in clinical outcome, perhaps because of the interference of other factors such as chemotherapy responsiveness or other yet unknown biological characteristics.

Our data seem to go against the original hypothesis that survivin might predict a more aggressive clinical outcome, although some evidences have been reported documenting the absence of any association between survivin expression and prognosis in some solid tumours (Grabowski et al, 2003; Li, 2003), and even a favourable prognostic role of high nuclear survivin content in gastric and bladder tumours (Okada et al, 2001; Lehner et al, 2002). The possibility that the biological and clinical role of survivin expression might also be influenced by tissue specificity cannot be ruled out and deserves further attention.

In vitro evidences showed that survivin might counteract chemotherapy-induced apoptosis (Zaffaroni et al, 2002), although univocal data have not been reported (Grossman et al, 2001; Pennati et al, 2002), possibly because of the use of different cell systems or the occurrence of peculiar surviving-cytotoxic drug 
interactions: for instance, survivin is able to bind polymerised microtubules through a putative tubulin-binding domain in the extended survivin C-terminal $\alpha$-helix (Verdecia et al, 2000), and counteract paclitaxel-induced apoptosis in NIH3T3 fibroblasts (Li et al, 1998). In clinical studies, Zaffaroni et al (2002) showed that high levels of survivin protein are associated with resistance to regimens containing the microtubule-targeting agent paclitaxel, but are unrelated to cisplatin responsiveness in advanced ovarian cancer. However, no data on the clinical relevance of this finding in terms of patient clinical outcome have been provided (Zaffaroni et al, 2002).

In the current series, which included patients with measurable disease at first surgery in order to give a better evaluation of chemotherapy response, we failed to find any association between cytoplasmic or nuclear survivin expression and response to chemotherapy, even after subgrouping patients administered platinum-based $v s$ paclitaxel-containing regimens. In addition, we could not find any difference in terms of TTP and OS according to either cytoplasmic or nuclear survivin status, in contrast with earlier studies which reported a negative prognostic role of survivin overexpression (Yoshida et al, 2001; Sui et al, 2002; Takai et al, 2002). However, it has to be taken into account that this is the first study examining the clinical relevance of survivin status in terms of clinical outcome in a single-institution large series of ovarian carcinomas, compared to previous reports which referred to very small sample series (Yoshida et al, 2001; Sui et al, 2002; Takai et al, 2002), sometimes biased by the inclusion of selected groups of patients (Yoshida et al, 2001).

Finally, the association between survivin, p53 and bcl-2 protein has been investigated based on the following background: (i) in the complex regulation of apoptosis and cell cycle progression, p53 and bcl-2 play a crucial role (Miyashita and Reed, 1993; Miyashita et al, 1994); (ii) wild-type p53 has been shown to negatively regulate human survivin at both mRNA and protein levels in 2774 ovarian carcinoma cells (Mirza et al, 2002), and to suppress survivin expression in lung adenocarcinoma cells (Hoffman et al, 2002); (iii) survivin expression has been associated with mutant p53 accumulation in ovarian and gastric cancer (Lu et al, 1998; Cohen et al, 2003), and during colorectal carcinogenesis (Kawasaki et al, 2001); moreover, a coassociation of survivin and bcl-2 has been found in breast and gastric cancer (Tanaka et al, 2000; Kawasaki et al, 2001). Our study, as well as other reports (Zaffaroni et al, 2002; Cohen et al, 2003), failed to show any relationship between p53 or bcl-2 and survivin expression, suggesting that these proteins could exert their functions through different mechanisms.

In conclusion, in our experience on a large series of patients, the immunohistochemical assessment of cytoplasmic and nuclear survivin status does not seem to be helpful in the prognostic characterisation of ovarian cancer. However, a more in depth investigation of the complex physiology of divergent survivin variants is needed in order to clarify the biological and possibly the clinical role of differentially located survivin isoforms.

Moreover, it is conceivable that, even though survivin has no prognostic role in ovarian cancer, it might be a potential target for apoptosis-based therapy, as testified by the increasing number of approaches aimed at (i) blocking survivin in cancer cells by small molecule antagonists, antisense oligonucleotides, ribozymes, dominant negative mutants (Reed and Wilson, 2003) or (ii) utilising survivin to create a tumour vaccine with dendritic cells (Pisarev et al, 2003; Reed and Wilson, 2003).

\section{ACKNOWLEDGEMENTS}

This work was partially supported by Associazione Italiana per la Ricerca sul Cancro (AIRC). 


\section{REFERENCES}

Altieri DC, Marchisio C (1999) Survivin apoptosis: an interloper between cell death and cell proliferation in cancer. Lab Invest 79: 1327 - 1333

Ambrosini G, Adida C, Altieri DC (1997) A novel anti-apoptosis gene, survivin, expressed in cancer and lymphoma. Nat Med 3: 917-921

Cohen C, Lohmann CM, Cotsonis G, Lawsin D, Santoianni R (2003) Survivin expression in ovarian carcinoma: correlation with apoptotic markers and prognosis. Mod Pathol 16: 574-583

Deveraux QL, Reed JC (1999) IAP family proteins - suppressors of apoptosis. Gene Dev 13: 239-252

Fanfani F, Ferrandina G, Corrado G, Fagotti A, Zakut H, Mancuso S, Scambia G (2003) Impact of interval debulking surgery on clinical outcome in primary unresectable FIGO Stage IIIc ovarian cancer patients. Oncology 65: 316-322

Ferlini C, Raspaglio G, Mozzetti S, Distefano M, Filippetti F, Martinelli E, Ferrandina G, Gallo D, Ranelletti FO, Scambia G (2003) Bcl-2 downregulation is a novel mechanism of paclitaxel resistance. Mol Pharmacol 64: $51-58$

Ferrandina G, Fagotti A, Salerno MG, Natali PG, Mottolese M, Maneschi F, De Pasqua A, Benedetti-Panici P, Mancuso S, Scambia G (1999) P53 overexpression is associated with cytoreduction and response to chemotherapy in ovarian cancer. Br J Cancer 81: 733-740

Fortugno P, Wall NR, Giodini A, O'Connor DS, Plescia J, Padgett KM, Tognin S, Marchisio PC, Altieri DC (2002) Survivin exists in immunochemically distinct subcellular pools and is involved in spindle microtubule function. J Cell Sci 115: 575-585

Grabowski P, Kuhnel T, Muhr-Wilkenshoff F, Heine B, Stein H, Hopfner M, Germer CT, Scherubl H (2003) Prognostic value of nuclear survivin expression in esophageal squamous cell carcinoma. Cancer Res 88: $115-119$

Grossman D, Kim PJ, Schechner JS, Altieri DC (2001) Inhibition of melanoma tumor growth in vivo by survivin targeting. Proc Natl Acad Sci USA 98: $635-640$

Hickman JA (1992) Apoptosis induced by anticancer drugs. Cancer Metast Rev 11: 121 - 139

Hoffman WH, Biade S, Zilfou JT, Chen J, Murphy M (2002) Transcriptional repression of the anti-apoptotic survivin gene by wild type p53. J Biol Chem 277: $3247-3257$

Hoskins WJ, Perez CA, Young RC (2000) Principles and Practice of Gynecologic Oncology pp 1005-1007. Philadelphia, PA: Lippincott Williams and Wilkins

Kaplan E, Meier P (1958) Non parametric estimation from incomplete observations. J Am Statist Assoc 53: 457-481

Kawasaki H, Toyoda M, Shinohara H, Okuda J, Watanabe J, Yamamoto T, Tanaka K, Tenjo T, Tanigawa N (2001) Expression of survivin correlates with apoptosis, proliferation and angiogenesis during human colorectal tumorigenesis. Cancer 91: 2026-2032

Lehner R, Lucia MS, Jarboe EA, Orlicki D, Shroyer AL, McGregor JA, Shroyer KR (2002) Immunohistochemical localization of the IAP protein surviving in bladder mucosa and transitional cell carcinoma. Appl Immunohistochem 10: 134-138

Li F, Ambrosini G, Chi EY, Plescia J, Tognin S, Marchisio PC, Altieri DC (1998) Control of apoptosis and mitotic spindle checkpoint by survivin. Nature 396: $580-584$

Li F (2003) Survivin study: what is the next wave? J Cell Physiol 197: 8-29

Lu CD, Altieri DC, Tanigawa N (1998) Expression of a novel antiapoptosis gene, survivin, correlated with tumor cell apoptosis and p53 accumulation in gastric carcinomas. Cancer Res 58: 1808-1812

Mahotka C, Liebmann J, Wenzel M, Suschek CV, Schmitt M, Gabbert HE, Gerhartz CD (2002) Differential subcellular localizaion of functionally divergent survivin splice variants. Cell Death Differ 9: 1334-1342
Mantel N (1966) Evaluation of survival data and two new rank order statistics arising in its consideration. Cancer Chemother Rep 50: $163-170$

Mirza A, McGuirk M, Hockenberry TN, Wu Q, Ashar H, Black S, Wen SF, Wang L, Kirschmeier P, Bishop WR, Nielsen LL, Pickett CB, Liu S (2002) Human survivin is negatively regulated by wild-type p53 and participates in p53-dependent apoptotic pathway. Oncogene 21: $2613-2622$

Miyashita T, Krajewski S, Krajewska M, Wang HG, Lin HK, Liebermann DA, Hoffman B, Reed JC (1994) Tumor suppressor p53 is a regulator of bcl-2 and bax gene expression in vitro and in vivo. Oncogene 9: $1799-1805$

Miyashita T, Reed JC (1993) Bcl-2 oncoprotein blocks chemotherapyinduced apoptosis in a human leukaemia cell line. Blood 81: $151-157$

O'Connor DS, Grossmann D, Plescia J, Li F, Zhang H, Villa A, Tognin S, Marchisio PC, Altieri DC (2000) Regulation of apoptosis at cell division by p34cdc2 phosphorylation of survivin. Proc Nat Acad Sci USA 97: $13103-13106$

Okada E, Murai Y, Matsui K, Isizawa S, Cheng C, Masuda M, Takano Y (2001) Survivin expression in tumor cell nuclei is predictive of a favourable prognosis in gastric cancer patients. Cancer Lett 163: 109-116

Olie RA, Simoes-Wust AP, Baumann B, Leech SH, Fabbro D, Stahel RA, Zangmeister-Wittke U (2000) A novel antisense oligonucleotide targeting survivin expression induces apoptosis and sensitises lung cancer cells to chemotherapy. Cancer Res 60: 2805-2809

Pennati M, Coltella G, Folini M, Citti L, Dandone MG, Zaffaroni M (2002) Ribozyme-mediated attenuation of survivin expression sensitizes human melanoma cells to cisplatin-induced apoptosis. J Clin Invest 109: $285-286$

Pisarev V, Yu B, Salup R, Sherman S, Altieri DC, Gabrilovich DI (2003) Fulllength dominant-negative survivin for cancer immunotherapy. Clin Cancer Res 9: 6523-6533

Reed JC, Wilson DB (2003) Cancer immunotherapy targeting survivin. Clin Cancer Res 9: 6310-6315

Salvesen GS, Duckett CS (2002) Apoptosis : IAP proteins: blocking the road to death's door. Nat Rev Mol Cell Biol 3: 401-410

Sui L, Dong Y, Ohno M, Watanabe Y, Sugimoto K, Tokuda M (2002) Survivin expression and its correlation with cell proliferation and prognosis in epithelial ovarian tumors. Int J Oncol 21: $315-320$

Takai N, Miyazaki T, Nishida M, Nasu K, Miyakawa I (2002) Expression of survivin is associated with malignant potential in epithelial ovarian carcinoma. Int J Mol Med 10: 211-216

Tanaka K, Iwamoto S, Gon G, Nohara T, Tenjo T, Tanigawa N (2000) Expression of survivin and its relationship to loss of apoptosis in breast carcinomas. Clin Cancer Res 6: $127-134$

Thompson CB (1995) Apoptosis in the pathogenesis and treatment of disease. Science 267: 1456-1462

Verdecia MA, Huang H, Dutil E, Kaiser DA, Hunter T, Noel JP (2000) Structure of the human anti-apoptotic protein survivin reveals a dimeric arrangement. Nat Struct Biol 7: 602-608

World Health Organization (1979) WHO Handbook for Reporting Results of Cancer Treatment. Vol. 48, pp 16-21. Geneva: WHO, Offset publication

Yoshida H, Ishiko O, Sumi T, Matsumoto Y, Ogita S (2001) Survivin, bcl-2 and matrix metalloproteinase- 2 enhance progression of clear cell- and serous-type ovarian carcinomas. Int I Oncol 19: 537-542

Zaffaroni N, Pennati M, Colella G, Perego P, Supino R, Gatti L, Pilotti S, Zunino F, Daidone MG (2002) Expression of the anti-apoptotic gene survivin correlates with taxol resistance in human ovarian cancer. Cell Mol Life Sci 59: 1406-1412 